\title{
A Critical Overview of Modelling and Simulating Methods for Complex Dynamic Systems
}

\author{
Peter P. Groumpos \\ Laboratory of Automation and Robotics, Department of Electrical and Computer Engineering, \\ University of Patras, Greece; *groumpos@ece.upatras.gr
}

SNE 27(4), 2017, 213 - 223, DOI: 10.11128/sne.27.on.10398 Received: December 15, 2017; Revised: December 18, 2017; Accepted: December 22, 2017

SNE - Simulation Notes Europe, ARGESIM Publisher Vienna, ISSN Print 2305-9974, Online 2306-0271, www.sne-journal.org

Abstract. Nowadays, practical dynamic systems have become more and more complex. The concept of Complex Dynamic Systems (CDS) arises in many scientific fields, technological areas and everyday's problems. The fundamental basics of complex dynamic systems are briefly presented and discussed. Modelling and simulating complex dynamic systems is a very difficult and challenging task. Different modelling approaches basically focus on the interaction between (microscopic) subsystems and the emergence of new qualities at the (macroscopic) system level. However these models are not sufficient to describe the dynamic behavior of today's systems. Similarly despite the numerous Simulation methods and large number of simulation software tools many of today's challenging problems cannot be studied appropriately and provide satisfactory answers. It seems that the uncertainty and fuzziness been inevitably present on these systems make the problems more difficult. The new models of Fuzzy Cognitive Maps are proposed as a new modelling approach for CDS. The need for new advanced models and new simulation methods is apparent taking into consideration the presence of human nature in all system processes, as well as the fuzzy and uncertaint environment. Some conclusions and future research directions are provided.

\section{Introduction}

In this overview paper the modeling and in parallel simulation methods been used in analyzing and studying today's Complex Dynamic Systems (CDS) is carefully, critically and wisely reviewed. The science of complex dynamical systems is a multidisciplinary field aiming at understanding the complex real world and its dynamic behavior that surrounds us.
Examples of these systems are: energy networks in distributed power systems with different renewable energy sources, neural networks in the brain that produce intelligence and consciousness, artificial intelligence systems, environmental systems, swarm of software agent, traffic patterns, biological systems, social and economic systems and many other scientific areas can be considered to fall into the realm of complex dynamical systems.

The title of the paper is very broad and overambitious to cover all issues of modelling and simulation methods for understanding the behavior of CDS. It is impossible to cover extensively and in a total exhaustive way the title of this paper. In order to accomplish this objective, we need an encyclopedia with many volumes.

The paper is rather provocative and by raising many challenging questions can be a sparkling fire that will drive the reader to unexpected avenues. Thus there are not given many details on most crucial and important issues. I leave the reader to search for the answers to the very important, critical, serious and challenging problems that the whole world is facing. There is an enormous literature with books, papers and many other sources that the reader should consult to find answers to the questions been raised in this paper.

Another very valuable and useful source for seeking answers is the International Journal SNE (Simulation Notes Europe). This SNE journal under the strong and enthusiastic editorial leadership of Felix Breitenecker and of the SNE Editorial Board, is an excellent source for following all the recent Research and Academic developments in the broad scientific area of Simulations. So once again I encourage the reader of this paper to focus on the questions been raised here and search for the new and innovative solutions that our academic and scientific communities so much are in need. 


\section{Challenging Issues in Modelling and Simulating Complex Dynamic Systems}

Modelling of a system is a fundamental work which is always the starting point for the understanding, designing, control, optimisation, and implementation of any physical and/or human made system. This is also the case for Complex Dynamic Systems (CDS). However, the last 30-40 years, Complex Dynamic Systems present many problems in mathematical modelling, control implementation, simulation methods and philosophical foundations. But why? What are the reasons? Do we need to be wiser? Certainly YES.

Complex Dynamic Systems (CDS) comprise of collections of many heterogeneous entities, which interact with other entities and their environment, which usually are having a lot of uncertainties, fuzziness, ambiguities and structural complexities. Interactions among subsystems are localized, often seeking autonomy and self-organizing, while most of the times are nonlinear, dynamic, fuzzy and possibly chaotic. The study of CDS represents a new approach to science that investigates how relationships between parts give rise to the collective behaviors of a system and how the system interacts and forms relationships with its environment. CDS have some specific characteristics, among which are: uniqueness, weak structuredness of knowledge about the system, incompleteness of its dynamic behavior, antagonism among different agents, the composite nature of system and heterogeneity of elements composing the complex system. Furthermore decisions must be made ensuring the smooth, reliable, stable and cost effective operation of each of the subsystem as well the whole CDS. There are many books on this modelling issue of Complex Dynamic Systems (CDS) [1]-[8].

Another important feature of CDS is that a network structure, including hierarchical one, self-organization can amount to:

(1) disconnecting certain constituent nodes from the system,

(2) connecting previously disconnected nodes to the same or to other nodes,

(3) acquiring new nodes,

(4) discarding existing nodes,

(5) acquiring new links,

(6) discarding existing links,

(7) removing or modifying existing links.
In addition today's society's challenging problems demand CDS to have a number of Properties-Abilities (P-A) such as: co-evolution, anticipation, adaptation, cooperation such as swarming, intelligence, consciousness, genetic regulation - homeostasis, development, disease, cascading failures in electrical grid, invasiveness in plants, hurricanes and self-repairing materials, cognition, emergence, self-evaluation and organization, robustness and wisdom.

All these collective dynamics of a CDS give rise to 'Emergent Evolution Properties-Abilities' (E.E.P-A) at higher scales in space and/or time. Under such conditions, the key problem of Complex Dynamic Systems and control theory consists in the development of methods of qualitative analysis of the dynamics and behavior of such systems and in the construction of efficient control algorithms for their efficient operation. In a general case, the purpose of control is to bring the system to a point of its phase space which corresponds to maximal or minimal value of the chosen efficiency criterion.

Another one of the main and actual problems in the theory of complex dynamical systems (CDS) and control sciences is a solution of 'ill-posed, weakly- and poorlystructured and weakly- formalizable complex problems' associated with complex technical, organizational, social, economic, cognitive and many other objects, and with the perspectives of their evolution. One more critical aspect that must be seriously taken into consideration is that the human presence in all CDS is inevitable. This problem is very critical in studying CDS because we are actually dealing with Dynamic Systems and want to understand their long-term qualitative behavior. However the focus is not on finding precise solutions to the equations, which most of the times are not well mathematically defining the complex dynamic system. Such a search is often hopeless.

The solutions been sought would rather answer questions like

- 'Will the CDS settle down to a steady state in the long term, and if so, what are the possible steady states?'

- 'Are the steady states, precise or are fuzzy and ambiguous?', or

- 'Does the long-term behavior of the system depend on its initial condition?' or

- 'How important is to depend on the knowledge of experts and if so, how many experts should be consulted?' or

- 'Does the past history of the behavior of the CDS influences its long-term behavior' or 
- 'What is more important: correlation or causality between the states?' or

- 'How sufficient are the mathematical models to predict the long term-behavior of the CDS?' or

- 'If not what other alternatives do we have?'.

A more fundamental question:

- 'Is there a difference between systems and mathematical models?'.

A system can be described in terms of its components and their interactions. A system is a group of related parts that make up a whole and can carry out functions its individual parts cannot. On the other hand, models are be used to represent systems and their interactions - such as inputs, processes and outputs - and energy and matter flows within systems. Systems may interact with other systems; they may have sub-systems and be a part of larger complex systems. On the other hand, models are limited in that they only represent certain aspects of the system under study. Thus, the crucial point in analyzing and studying the dynamic behavior of complex systems is to clearly understand this fundamental difference, and analyze and study the different modelling approaches for CDS.

\section{An Overview of Mathematical Approaches for Modelling CDS}

The issue of mathematical modelling of CDS is not an easy task. In addition, many times the difference between the system and its mathematical representation of a process or operation of a complex system is not clear. This is very critical. Systems and System Models are useful in science and engineering because the world is complex, so it is helpful to isolate a single system and construct a simplified model of it. In order to do this, scientists and engineers imagine an artificial boundary between the system in question and everything else. They then examine the system in detail and using a variety of modelling methods a mathematical model is generated.

It is different to model a physical or human made system with Ordinary Differential Equations (ODE) than to represent its operation through for example with a Taylor series approximation model. Some would argue that most models are often an approximation of the physical properties and dynamic characteristics of a system. This is partially true however, the validity of the classification is limited to the accuracy with which the particular model is described and moreover the way is "behaving" in the real world. In general systems are classified according to the mathematical representation (equations or not) used to describe them and how they are prepared. It is not the objective of this paper to discuss extensively all types of classifications with all their ramifications.

Rather we will single out that type of systems, which are within the scope of the title especially with respect to simulation modelling and analysis. Furthermore the classical classification just 60-70 years ago, started with continuous vs. discrete systems. This to me is a very important issue. I argue that the classification of systems is different than the mathematical model representation of the corresponding system. I believe it was wrong to start this way the classification. I believe that the first classification is deterministic vs. stochastic models. In deterministic models, the output of the model is fully determined by the parameter values and the initial conditions. Stochastic models possess some inherent randomness. The same set of parameter values and initial conditions will lead to an ensemble of different outputs. The next classification is linear vs. nonlinear systems. The next classification static vs. dynamic systems. Then continuous vs. discrete systems and then, time varying vs. time invariant.

The important remark here is that for each system classification there should be a similar modelling classification. However this is not always the case and easy to be done. For example a system is non linear however the mathematical model could be a linear approximation. This further complicates the appropriate simulation model and software tool that should be used to simulate the dynamic behavior of the system.

\section{Basics of Simulation M odelling and Analysis}

Simulation is much more meaningful when we understand what it is actually doing. Understanding how simulation works helps us to know whether we are applying it correctly and what the output results mean. But what is simulation? And what is its relation to modelling? Simple and easy questions but difficult to comprehend the answers to them and more important to understand how to use both (modelling and simulation) to analyze and understand the behavior of Complex Dynamic Systems (CDS). Many books have been written that give thorough and detailed discussions of the science of simulation [9]- 
[14]. Let enlighten ourselves with a few definitions and methods for Simulations. To simulate is to try to duplicate the characteristics of a real system and to approximate its dynamic behavior Simulation is one of the most widely used decision modeling techniques. Before the coming of the digital age, only analog computers existed and analog simulations were developed and used. In other words, till digital simulations came to the academic and scientific communities after the 1950ies.

In order to understand the significant difference and the tremendous push that the digital computer provided to the simulations a few examples of analog computers and their applications should be mentioned. The technology of the ubiquitous operational amplifier - now an indispensable component of every control system - was originally invented and perfected for analog computing. Columbia professor John Ragazzini invented the term "operational amplifier" and described the state-of-the-art electronic analog techniques in his landmark paper [15].

The simulation of guided missiles in the late 1950ies pushed the limits of both analog and digital computing. Digital computers were not yet fast enough to permit hardware-in-the-loop testing of the real-time control systems, but analog computers did not have the accuracy or dynamic range to simulate the long-range trajectories. Electronic analog-to-digital converters, another now-indispensable technology, were first commercialized for the purpose of interfacing analog computers with digital machines. These hybrid computers bridged the gap, using analog techniques to simulate the vehicle with its control surfaces and using digital techniques to calculate navigational coordinates.

As a tool, the early differential analyzer was more than a general-purpose, differential-equation solver. It was an educational tool and a research touchstone. The history of control is entwined with the history of analog computing. Many of the tools, technologies, and theories of control were enabled by, or are directly descended from, mechanical and electronic analog computers. Ana$\log$ computers played a key role in enabling the simulation of control systems for several decades, leading to a better understanding of theory and better designs in practice.

Today Simulation imitates the operations of a system or process, usually via computers. We must emphasize: what's being simulated is the system, figure 1 . To study the system, often make assumptions/approximations, both logical and mathematical, about how it works.

These assumptions form a model of the system. If model structure is simple enough, could use mathematical methods to get exact information on questions of interest - analytical solution.

But most complex systems require models that are also complex in order to be closer to the real system. Thus, it must be studied via simulation - evaluate model numerically and collect data to estimate model characteristics and subsequently to understand the dynamic (or static) behavior of the system.

\section{Modelling and Simulation Methods for Complex Dynamic Systems}

As was explained above simulation models or methods can be developed to study the behavior of Complex systems. However the task is very complicated because all scientific areas have CDS. Even more difficult is the task, to approach each thematic area and perform the modelling and simulation task.

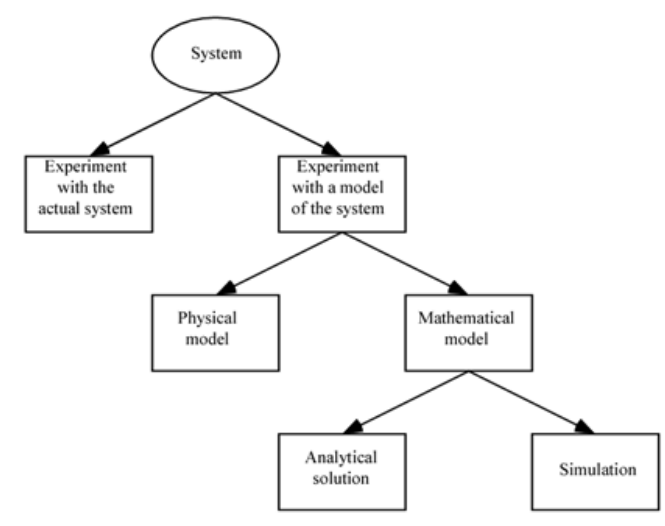

Figure 1: A flow chart for addressing the steps of modeling and simulation of CDS.

So for different complex scientific areas a good number of mathematical models and the corresponding software tools have been developed. Let us see some of them.

ENGINEERING SYSTEMS: for these systems the following four (4) generic mathematical approaches for modelling any CDS are proposed:

1) the Ordinary Differential Equations

2) the impulse function response

3 ) the transformation methods and

4) the state space representation. 
The main and very important point here is that all four generic approaches are modelling exactly the same physical or human made system. Knowing the one all the other three can be generated. This, four (4) generic mathematical approaches in modelling the same complex system is presented for first time here. However, for the purpose of this paper we will review in the more classical ways the modelling problem of CDS.

The focus is on modeling techniques that can glue together subsystems from diverse physical domains. Thus it depends also on the sub-scientific area that we are trying to solve a problem. For example, the concept of energy (or power), which is universally valid across many physical domains, is the right tool for combining electrical, mechanical, hydraulic, pneumatic, thermal and thermodynamic systems. However, some of the developed methods using the concept of energy is at least partially useful in the domains where the concept of energy is not so useful such as socio-economic systems. In total three groups of modeling techniques, which are based on the concept of energy have been develope. [2], [4], [8].

Analytical methods based on the Lagrangean and Hamiltonian functions well known from the studies in theoretical physics and/or mechanics, object-oriented modeling as an alternative to the more widespread blockoriented modeling, and last but not least an intuitive graphical technique known as bond graph modeling. Whichever methodology is followed to create the mathematical model, of the ways to analyze it is a numerical simulation, that is, numerical solution of the corresponding differential or differential-algebraic equations. Then appropriate simulation tools will be developed on the basics of numerical techniques for differential and differential-algebraic equations with the objective to understand the basic issues such as approximation errors, numerical stability and suitability of the common methods for different classes of models.

Thus the task of modelling and simulation methods for CDS is not a simple task. There have been developed many simulation models for engineering complex systems. We can have mentioned here the two most common simulation tools: MATLAB and Simulink.

MATLAB is the high-level language and interactive environment used by millions of engineers and scientists worldwide. It lets you visualize ideas across disciplines including signal and image processing, communications, control systems, and computational finance. Simulink is a block diagram environment for multi-domain simula- tion and Model-Based Design. It supports simulation, automatic code generation, and continuous test and verification of embedded systems. Simulate dynamic systems leveraging graphical editors, customizable block libraries, and solvers for modeling. An important remark is that when you use MATLAB and Simulink together, you combine textual and graphical programming to design a more powerful simulation software tool. The interesting reader can find many useful materials for both simulation software tools.

BUSINESS AND ECONOMICS: Business and Economic sectors there are many models and software tools. One of the most known simulation model is the Monte Carlo Simulation model. For historical reasons it was developed during the Second World War. Before the Monte Carlo method was developed, simulations tested a previously understood deterministic problem and statistical sampling was used to estimate uncertainties in the simulations. Monte Carlo simulations invert this approach, solving deterministic problems using a probabilistic ana$\log$ and solving the problem probabilistically. These simulations were used in investigating radiation shielding and the distance that neutrons would likely travel through various materials.

They were a vital simulation in the Manhattan Project, (of the atomic bomb), even though the computational tools were underdeveloped. During their work on the Manhattan Project John von Neumann and Stanislaw Ulam named the method after the Monte Carlo Casino in Monaco. Just for the Monte Carlo Simulation approach quite a few software tools have been developed: GoldSim, TRIDYN, Fluka, Maestro, PSAT, C++ to mention a few and just for one simulation approach. Please also note that the software to be used depends on the application working on.

MEDICAL SYSTEMS: Simulation enables healthcare systems and processes to be tested in a safe, virtual environment without risk to patients and staff - supporting organizations to make better decisions, improve systems of care and maximize resources. There are simulation tools: SIMUL8. PSF, Apollo, CSSC (for clinical studies), UroSim, LapSim (Laparoscopy), FlexSim, VisualSim (for ophthalmology) and many others.

IMPORTANT REMARK: it is impossible to cover extensively and in a total exhaustive way the title of this paper. In order to accomplish this objective, an encyclopedia with many 'scientists' and many volumes is 
needed. So once again I encourage the reader of this paper to focus on the questions. The author trying the above analysis believes that he contributes a small piece of scientific work, aiming in developing a new uniform theory of Intelligent and Cognitive Systems theory. Such a new theory is badly needed as will be explained below.

\section{Do We Need New Mathematical Modelling and Simulation Tools for CDS?}

In the previous sections we have seen that numerous mathematical modelling approaches and similarly a large number of simulation software tools for the Complex Dynamic Systems have been developed. However, we are facing every day numerous unsolved, critical and challenging problems. In Section 1, many questions where raised regarding the issues of modelling of CDS.

ONE VARY BASIC QUESTION IS: ARE WE HAPPY WITH TODAYS' MATHEMATICAL MODELLING AND SIMULATING APPROACHES?

If yes then why we keep failing to solve the basic problems of our societies? The poor children of the third world countries do not have clean drinking water! Even worst: we are not capable to solve the many problems our world is facing but instead with our actions we generate even more problems, which further complicate the whole problem. Our ignorance and/or our denial to realize the threats that we ourselves create with our actions, to preserve our environment. We are the ones that waste the energy sources foolishly and/or do not utilize better the renewable energy sources (RES).

If we are not happy what are we doing to correct our past mistakes? Have we learned from history or do we insist to ignore it?

More questions can be raised which are relevant to this paper. Do all today's models and associated solutions provide satisfactory and working conditions to the everyday behavior of Complex Dynamic Systems?

Indeed, there is a good and large number of situations or problems where today's models and solutions fail to give satisfactory answers. Making decisions in the area of Complex Dynamic Systems often strains our cognitive capabilities. Uncertainty and related concepts such as risk, fuzziness and ambiguity are prominent in the research and accompanied literature on Decision-Making.
- What are the best models?

- Do all models have detailed software tools that can adequately simulate their behavior?

- What is intelligence?

- What is wisdom?

- Is Intelligent Control related to Cognitive Control?

- Do all today's models and associated solutions provide satisfactory and working conditions to the everyday behavior of Complex Dynamic Systems?

Indeed, there is a good and large number of situations or problems where today's models and solutions fail to give satisfactory answers. Where have we failed? I believe that our weakness lies in:

- a) Our inability to comprehend and understand well and precisely the actual dynamic and chaotic behavior of CDS in the presence of uncertainty, fuzziness and structural complexity and

- b) due to uncertainty, fuzziness and structural complexity we have different interpretations and mathematical explanations by different people for the same real physical system.

Are we happy? Certainly not. Omnipresent in realistic settings, uncertainty and fuzziness constitutes a major obstacle to modeling and effectively analyzing Complex Dynamic Systems. How do we utilize knowledge and learning? Do we need new advance, innovative approaches? There appears to be a lack of new ideas in driver behavior modeling. Although behavioral research is under some pressure, it seems too facile to attribute this deplorable state of affairs only to a lack of research funds.

In my opinion the causal chain may well run in the opposite direction. An analysis of what is wrong has led me to the conclusion that human factors research in the area of driver behavior has hardly been touched by the 'cognitive revolution' that swept psychology in the past fifteen years. A more cognitive approach might seem advisable and the 'promise of progress' of such an approach should be assessed. Such an approach is provided with the advanced Fuzzy Cognitive Maps. This topic is addressed next.

\section{Modelling Using Fuzzy Cognitive Maps}

Fuzzy Cognitive Maps came as a combination of the methods of fuzzy logic and neural networks was first introduced by Kosko [16] only 30 years ago. A detailed presentation of FCM is provided in [18]. 
They constitute a computational method that is able to examine situations during which the human thinking process involves fuzzy or uncertain descriptions. An FCM presents a graphical representation used to describe the cause and effect relations between nodes, thus giving us the opportunity to describe the behavior of a system in a simple and symbolic way. In order to ensure the operation of the system.

FCMs embody the accumulated knowledge and experience from experts who know how the system behaves in different circumstances. This knowledge is extracted using linguistic variables which then are transformed to numeric values using a defuzzification method. In other words, they recommend a modeling process consisting of an array of interconnected and interdependent nodes $C_{i}$ (variables), as well as the relationships between them $\mathrm{W}$ (weights). Concepts take values in the interval $[0,1]$ and weights belong in the interval $[-1,1]$. Fig.2 shows a representative diagram of a FCM.

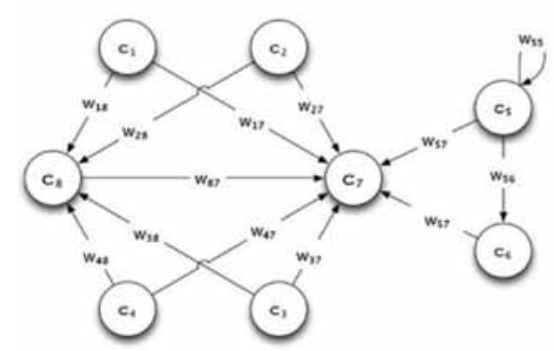

Figure 2: Fuzzy Cognitive Map.

The full procedure of the development of a FCM follows the below steps:

- $\quad$ Step 1: Experts select the number and the kind of concepts $C_{i}$ that constitute the Fuzzy Cognitive Map

- Step 2: Each expert defines the relationship between the concepts

- Step 3: They define the kind and the value of the relationship between the two nodes

- Step 4: Experts describe the existing relationship firstly as 'negative' or 'positive' and secondly, as a degree of influence using a linguistic variable, such as 'low', 'medium', 'high' etc.

The sign of each weight represents the type of influence between concepts. There are three types of interconnections between two concepts $C_{i}$ and $C_{j}$ :

- $\quad w_{i j}>0$, an increase or decrease in $C_{i}$ causes the same result in concept $C_{j}$.
- $\quad w_{i j}<0$, an increase or decrease in $C_{i}$ causes the opposite result in $C_{j}$.

- $w_{i j}=0$, there is no interaction between concepts $C_{i}$ and $C_{j}$.

The degree of influence between the two concepts is indicated by the absolute value of $w_{i j}$. During the simulation the value of each concept is calculated using the following rule:

$$
A_{i}(t)=f\left(k_{1} A_{i}(t-1)+k_{2} \sum_{\substack{j=1 \\ j \neq i}}^{n} A_{j}(t-1) w_{j i}\right)
$$

Where $\mathrm{t}$ represents time, $\mathrm{n}$ is the number of concepts and $\mathrm{f}$ is the sigmoid function given by the following equation:

$$
f=\frac{1}{1+e^{-\lambda x}}
$$

Where $\lambda>0$ determines the steepness of function $f$. The FCM's concepts are given some initial values which are then changed depending on the weights; the way the concepts affect each other. The calculations stop when a steady state is achieved, the concepts' values become stable. A more comprehensive mathematical presentation of FCMs with application to real problems with very useful results is provided in [17],[19]-[29].

There have been many efforts for the evolution of FCM over the last few years, but Simulation software tools are still missing.

\section{Drawbacks of Fuzzy Cognitive Maps}

In the previous sections trying to answer all challenging problems and questions in modelling and simulating CDS, FCMs were proposed as a new alternative and innovative approach to deal with these fundamental issues. The mathematical presentation along with the two examples from real problems give the academic and scientific communities hopes for overcoming some of the problems been encountered in modelling and simulating CDS using the classical approaches

However, with the current modeling of FCMs and their extensive use in solving many real problems and applications, various interesting and challenging problems and drawbacks have emerged. The early hope and enthusiasm that FCM would be a strong and effective approach to be able to solve the difficult problems of the Complex Dynamic Systems. However, FCMs theories are around only less than 30 years. 
We should not give up now. We must re-address the basic fundamentals that drove the scientific and academic community to develop FCMs, while at the same time keeping the core of the 'initial philosophy and methodology' intact. Artificial Intelligence (AI) has been around more than 70 years and despite the skepticism of some well known scientists AI still continues strong on funding and research efforts.

Prof Stephen Hawking, one of Britain's pre-eminent scientists, has said that efforts to create thinking machines pose a threat to our very existence. He told in an interview the BBC: 'The development of full artificial intelligence could spell the end of the human race.' His warning came in response to a question about a revamp of the technology he uses to communicate, which involves a basic form of AI. The theoretical physicist, who has the motor neurone disease amyotrophic lateral sclerosis (ALS), is using a new system developed by Intel to speak using AI. Prof Hawking is not alone in fearing for the future. In the short term, there are concerns that clever machines capable of undertaking tasks done by humans until now will swiftly destroy millions of jobs.

In the longer term, the technology entrepreneur Elon Musk has warned that AI is "our biggest existential threat". Many believe that AI is our biggest scientific mistake especially if it is left to scientists and politicians that do not have the human beeing as the center of our activities and/or they do not respect human values.

Therefore re-addressing the FCMs we should be careful and wise. Let us restate what is a FCM and why we claim that is a promising and innovative method for studying CDS

A Fuzzy Cognitive Map (FCM) provided a way to identify the most important structural elements in modeling and controlling Complex Dynamic Systems (CDS). Complete, efficient and practical mechanisms to analyze and predict the evolution of data been fuzzy, incomplete, and vague in CDS were not available for years due to several reasons. Numerical data for years have been considered as crisp and exact values.

However today most data may be fuzzy, uncertain or hard to come by, and the formulation of a precise mathematical model may be difficult, costly or even impossible. Then efforts to introduce knowledge on these systems should rely on natural language arguments and the human intervention in the absence of formal models.

However, although very efficient and simple to use, FCM are causal maps (a subset of cognitive maps that only allow basic symmetric and monotonic causal relations between concepts), and, in most applications, avoiding the need to use extensive and time consuming differential equation models, while obtaining very interesting and encouraging results. By using true qualitative modelling techniques, FCM obtained results that look more realistic (plausible) than those obtained using quantitative approaches - where results almost never show the short term uncertainties that are so characteristic of qualitative real-world dynamic systems. In the end, the results of the FCM model and all related applications, that were developed more than 25 years ago, are surprisingly realistic and could have been used to predict and avoid the current world economic crisis, even if one considers its necessary incompleteness.

An FCM is a qualitative mathematical tool rather than a quantitative tool. It provides a simple, flexible and straightforward approach to model the dynamic behavior of a complex system, which is composed of various components or subsystems. An FCM can always describe any CDS using a mathematical model with the following six (6) characteristics or attributes:

1) Defined causality indicating positive or negative relationship between all components

2) The causal links are always dynamic and never static

3) Past knowledge of the CDS dynamic behavior is available and reliable

4) Human-like reasoning and

5) Always availability of experts knowing the dynamic behavior of the CDS.

Given that the above hold and the FCM methodologies, so far been developed, we can model any given CDS. Solutions to this drawback were also provided there. There also other drawbacks of FCM that need to be addressed and been solved. These solutions are going to overcome some of these limitations and offer more accurate results and a better view and knowledge of the CDS.

One major drawback that has been raised by the author of this article is that concepts of an FCM include everything: states, inputs, outputs, constraints and all other parameters which are going to be examined regardless their nature [29]. However this is not mathematically correct and logical in any scientific approach. Why, for example in classic FCM theories the fact that some concepts are not being affected by others thus they have to stay static through the whole iteration process. 
However due to the current approach, Eq.1 and Eq.2 their value changes after the first iteration which is not correct. In addition having all variables in one 'concept vector' the iteration step $\mathrm{k}$ in Eq.1, is the same for all concepts which is also not true in real problems and mathematically not correct. Why must the inputs and outputs of the CDS change at every iteration step $\mathrm{k}$ ?

For example, on a health treatment of a patient, why must the inputs (concepts) (e.g. the medication dose of a drug that is given every morning) and the outputs (e.g. the blood test results (concepts) that are monitored every two or three days) be changed every time the 'health conditions' (concepts) of the patient is monitored every second or every hour? However, this is the case using classic FCM theories especially using equations 1 and 2.

Even the calculation method of the values of the concepts, (Eq.1) has a serious drawback. The calculation equation takes into consideration the change that each concept cause separately instead of the total change which is caused to the concept $C_{i}$. This results in a large increase to the value of the concept $C_{i}$ that goes far beyond the interval $[0,1]$. This is the reason why the sigmoid function (Eq.2) is needed; to suppress the result to the interval $[0,1]$.

However due to the shape of the sigmoid curve any concept value beyond 3 leads the sigmoid function to correspond it to the value 1 which is greatly problematic as the final output is corresponded to the linguistic variable 'high' even if this is not always the expected or correct result. However continuing on the subject of the sigmoid function, there is another drawback that leads to high output values. This is the fact that the center of the sigmoid curve instead of being on the $(0,0)$ point on the $x y$ axis it is on the $(0.5,0)$ point. This means that each concept's lowest value can be the 0.5 . This problem combined with the first one makes it difficult to interpret the obtained results.

Continuing with the NHL learning method, while running several simulations we have observed that due to the way weights are being calculated if the number of iterations of the algorithm is increased, in order to reach a steady state, the causality reverses and all or some of the $w_{i j}$ become positive. This is a very serious drawback as it changes the causality between concepts and in several occasions instead of having a lower we are going to have a larger result which can cause serious problems not only in the interpretation of the obtained results but also on stability issues to a number of systems.
There are a number of other drawbacks that need to be addressed but this should be the work of future papers. However for the above raised drawbacks some solutions and explanations must be given. The research team of the Laboratory for Automation and Robotics under the supervision of the author of this paper have provided some interesting and valuable solutions.

As it was mentioned, above in this section, in the classic FCM representation ALL the concepts are ALL the parameters which are going to be examined regardless their nature. However, in a CDS, even when it is described in a fuzzy way through an FCM the main concept is the same.

Each system has its states, inputs, outputs and other parameters and constraints. However, since an FCM is a representation of such a system, this fundamental characteristic should be taken into consideration. For this reason, as in the classic control theory methods [2]-[4], [7][8], the concepts of a Fuzzy Cognitive Maps are separated into the following three categories:

A) Fuzzy State Concepts: The concepts describing the dynamic operation of the system, $\mathrm{x}$.

B) Fuzzy Input Concepts: The inputs of the system, $u$ and

C) Fuzzy Output Concepts: The concepts describing the outputs of the system, $y$

In this way a better knowledge of the dynamic behavior of the CDS is gained. The proposed separation facilitates not only the understanding of the system's operation but also the calculation of the concepts' values in their physical nature as the states, inputs and outputs of the real system.

Authors have proposed a new calculation rule. The two equations extracted from the classic FCM are the followings:

$$
\begin{aligned}
x_{k+1} & =f\left[A x_{k}+B u_{k}\right] \\
y_{k} & =f\left[C x_{k}+D u_{k}\right]
\end{aligned}
$$

where $x_{k} \in \mathrm{R}^{\mathrm{n}}$ is a state vector, $u_{k} \in \mathrm{R}^{\mathrm{r}}$ is an exogenous known input vector, $y_{k} \in \mathrm{R}^{\mathrm{m}}$ is the output vector and $f$ is an activation function. The new model was implemented for first time in diagnosing meniscus injury in the IFAC World Congress 2017 with very encouraging results [29]. 


\section{Conclusions and Future Research}

In this overview paper one of the most difficult and challenging problems in modelling, analyzing and simulating Complex Dynamic Systems (CDS) has been seriously addressed. The analysis and efficient control of CDS are impossible without a formal model of the system. However, today's technologies for building such models for CDS are not sufficient. Then the necessary simulations cannot describe the dynamic behavior of the system under study. Qualitative description of most of the parameters of Complex Dynamic Systems results inevitably in fuzziness, complexity and uncertainty.

Thus the human cognition and presence is absent from the modelling band simulation approaches. New approaches are needed. Fuzzy Cognitive Maps seem to provide a possible solution. However, they do have quite a few drawbacks that must be resolved.

\section{Challenging future research directions? Just take} each question been raised in this paper and you have a big number of research challenges:

- new models of FCMs for CDS using learning methods;

- develop new simulation models and software tools using intelligent systems and advanced neural network theories;

- develop mathematical models using new advance FCMs for different applications and using a number of experts;

- develop new software tools for various CDS and perform extensive simulations, and

- develop a new Intelligent and Cognitive Sytems Control theory using the human as the center of such an effort [30].

\section{References}

[1] Luenberger D G. Introduction to dynamic systems: theory, models, and applications. Wiley, 1979.

[2] Beltrami E J. Mathematics for Dynamic Modeling, Vol 2. Academic Press, 1998

[3] Sandquist G M.Introduction to System Science.Prentice Hall, Inc. Englewood. Cliffs, N.J. 1985.

[4] Bar-Yam Y. Dynamics of Complex Systems. Reading, MA: Addison-Wesley, 1997.
[5] Mesarovic M, Macko D, Takahara Y. Theory of hierarchical multilevel systems. New York: Academic Press, 1970.

[6] Levin S A. Complex adaptive systems: Exploring the known, the unknown and the unknowable. Bulletin of the American Mathematical Society. 2003; 40:3-19.

[7] Nelson R J. Structure of Complex Systems. Philosophy of Science Association. 1976; 2:523-542.

[8] Ottino J M. Engineering complex systems. Nature, 2004; 427: 399-399.

[9] Banks J (editor). Handbook of Simulation: Principles, Methodology, Advances, Applications, and Practice. (1st Edition), Engineering and Management Press (EMP), 2001

[10] Pooch U D, Wall J A. Discrete Event Simulation: A Practical Approach. Cre Press Computer Engineering Series, Mishawaka, IN, U.S.A, 1993.

[11] Hannon R E. Systems simulation: the art and science. Prentice-Hall, 1975.

[12] Widman L E, Loparo K A, Nielsen N R. Artificial Intelligence, Simulation and Modeling. Wilye Interscience, N.Y. 1989.

[13] Law A. Simulation Modeling and Analysis , McGraw-Hill Series in Industrial Engineering and Management. 5th Edition, 2013.

[14] Thesen A, Travis L. E. Simulation for Decision Making. West Group,1992.

[15] Ragazzini J R, et al. Analysis of problems in dynamics by electronic circuits. Proceedings of the IRE, vol. 35. p. 444, May 1947.

[16] Kosko B. Fuzzy cognitive maps. International Journal of man-machine studies. 1986; 24(1):65-75.

[17] Groumpos P P, Stylios C D. Modeling supervisory control systems using fuzzy cognitive maps. Chaos Solitons \& Fractals. 2000; 113: 329-336.

[18] Groumpos P P. Fuzzy Cognitive Maps: Basic Theories and their Application to Complex Systems. Glykas M. (ed) Fuzzy Cognitive Maps: Advances in Theory, Methodologies, Tools and Applications. Springer-Verlag Berlin, Heidelberg, 2010; 247: 1-22.

[19] Papageorgiou E I, Stylios C D, Groumpos P P. Active Hebbian learning algorithm to train fuzzy cognitive maps. International Journal of Approximate Reasoning, 2004;37: 219-249.

[20] Groumpos P P, Anninou A P. A theoretical mathematical modeling of Parkinson's disease using Fuzzy Cognitive Maps. 12th International Conference on Bioinformatics and Bioengineering (BIBE 2012), pp 677 - 682.

[21] Taber W R. Knowledge processing with fuzzy cognitive maps. Expert Systems with Applications, 1991; 2(1): 83-87. 
[22] Lee S, Ahn H. Fuzzy cognitive map based on structural equation modeling for the design of controls in businessto-consumer e-commerce web-based systems. Expert Systems with Applications. 2009; 36(7): 10447-10460

[23] Stylios C D, Groumpos P P. Modeling complex systems using fuzzy cognitive maps. IEEE Transactions on Systems, Man and Cybernetics, Part A: Systems and Humans. 2004; 34(1):155-162.

[24] Carmona M A, Barbancho J, Larios D F, León C. Applying case based reasoning for prioritizing areas of business management. Expert Systems with Applications. 2013; 40(9):3450-3458.

[25] Carmona M A, Barbancho J, Larios D F, León C. Applying case based reasoning for prioritizing areas of business management. II, Expert Systems with Applications. 2013; 40(9):3450-3458.

[26] Anninou A P, Groumpos P P, Polychronopoulos P. Modeling Health Diseases Using Competitive Fuzzy Cognitive Maps. Artificial Intelligence Applications and Innovations. Springer Berlin Heidelberg, 2013, pp.88-95
[27] Bourgani E, Stylios C D, Manis G, Georgopoulos V C. Time dependent fuzzy cognitive maps for medical diagnosis. In Hellenic Conf. on AI, 544-554, Springer, 2014.

[28] Carvalho J P, Tome J. Issues on the stability of fuzzy cognitive maps and rule-based fuzzy cognitive maps. In Proceedings of the 21st International Conference of the North American Fuzzy Information Processing Society, NAFIPS2002, New Orleans, 2002.

[29] Anninou A. P, Groumpos P P, Poulios P, Gkliatis I. A New Approach of Dynamic Fuzzy Cognitive Knowledge Networks in Modelling Diagnosing Process of Meniscus Injury”. IFAC-PapesOnLine, 2017, 50(1), 5861-5866.

[30] Groumpos P P. Intelligent Control and Cognitive Control: Issues and Challenges. In Brain Function Assessment in Learning, First Intern. Conf. BFAL, Proc. by Springer pp. 1-3, Patras, Greece, September 2017. 